\title{
Hidden Markov modelling of spike propagation from interictal MEG data
}

\author{
A Ossadtchi ${ }^{1}$, J C Mosher ${ }^{2}, \mathrm{~W}$ W Sutherling ${ }^{3}, \mathbf{R}$ E Greenblatt ${ }^{1}$ \\ and $R$ M Leahy ${ }^{4}$ \\ ${ }^{1}$ Source Signal Imaging Inc., San Diego, CA 92103, USA \\ 2 Biological \& Quantum Physics Group, Los Alamos National Laboratory, Los Alamos, \\ NM 87545, USA \\ ${ }^{3}$ Epilepsy and Brain Mapping Program, Huntington Memorial Hospital, Pasadena, \\ CA 91105, USA \\ ${ }^{4}$ Signal and Image Processing Insitiute, USC, Los Angeles, CA 90089, USA \\ E-mail: leahy@sipi.usc.edu
}

Received 17 February 2005, in final form 12 May 2005

Published 6 July 2005

Online at stacks.iop.org/PMB/50/3447

\begin{abstract}
For patients with partial epilepsy, automatic spike detection techniques applied to interictal MEG data often discover several potentially epileptogenic brain regions. An important determination in treatment planning is which of these detected regions are most likely to be the primary sources of epileptogenic activity. Analysis of the patterns of propagation activity between the detected regions may allow for detection of these primary epileptic foci. We describe the use of hidden Markov models (HMM) for estimation of the propagation patterns between several spiking regions from interictal MEG data. Analysis of the estimated transition probability matrix allows us to make inferences regarding the propagation pattern of the abnormal activity and determine the most likely region of its origin. The proposed HMM paradigm allows for a simple incorporation of the spike detector specificity and sensitivity characteristics. We develop bounds on performance for the case of perfect detection. We also apply the technique to simulated data sets in order to study the robustness of the method to the non-ideal specificity-sensitivity characteristics of the event detectors and compare results with the lower bounds. Our study demonstrates robustness of the proposed technique to event detection errors. We conclude with an example of the application of this method to a single patient.
\end{abstract}

(Some figures in this article are in colour only in the electronic version)

\section{Introduction}

Epilepsy is a family of brain disorders characterized by seizures that result in disturbances of perception, behaviour and consciousness. Using electro- or magnetoencephalography (EEG 
or MEG, or collectively EMEG), it is possible to record interictal activity, or spikes, during the intervals between seizures, i.e. the interictal periods. It is believed that these spikes originate from the same brain foci as do the seizures (Diekmann et al 1998). Spike onset focus determination using EMEG data and source localization methods can be an important component in the presurgical evaluation of patients for whom pharmacotherapy has not been successful in controlling seizures, and may reduce the need for invasive diagnostic procedures, such as depth recordings.

Recent work has demonstrated that the problem of epileptogenic zone detection from extracranial recordings is more complex than was thought originally. Manual analysis of interictal data sets (spike identification followed by dipole fitting) (Diekmann et al 1998) often yields several dipole clusters within the brain. Automatic spike detection techniques (Ossadtchi et al 2004, Kobayashi et al 2002) for the analysis of interictal MEG/EEG data sets also yield several potentially epileptogenic regions in the brains of patients with partial epilepsy.

The presence of multiple epileptogenic zones may be explained by propagation of the interictal activity between different parts of the brain. In a study of five patients with temporal lobe epilepsy, Sutherling and Barth (1989) found propagation of interictal activity between deep and superficial cortex areas of the temporal lobe. Propagation times were estimated to be on the order of 30-35 ms, consistent with axonal conduction velocities. Another study (Baumgatner et al 1995) reported propagation of interictal activity between mesiobasal temporal lobe and lateral temporal neocortex areas. In Kobayashi et al (1999), interictal spikes of slightly different latencies were observed as independent components with distinct topographies, consistent with propagation of interictal activity between different areas of the brain. These studies lead to the conclusion that propagation analysis can be an important component in identifying the primary epileptogenic focus from interictal activity. Bourien et al (2004) have found that taking propagation patterns into consideration significantly improves the surgical outcome and reduces the likelihood of surgery-induced deficit.

Patterns of propagation activity can be established based on the analysis of either ictal or interictal recordings. Ictal activity has been studied using imaging (PET and fMRI), as well as electrophysiological methods, both invasive (electrocorticography and depth recordings) and non-invasive (EMEG), applying functional connectivity measures, such as coherence and phase synchrony for propagation detection. Early efforts using fMRI and PET data were based on linear coherence measures and employed canonical variates analysis (CVA) (Strother et al 1996) and partial least squares (PLS) (McIntosh et al 1996) approaches. However, the temporal resolution of the underlying imaging modalities (fMRI and PET) only allows the detection of coupling on the time scale of seconds, significantly slower than the observed electrophysiological time scale. In addition, CV and PLS techniques can only detect a linear coupling and are limited to assessing pairwise interactions.

Other functional connectivity measures that overcome some of these limitations have been applied to the analysis of experimental invasive recordings. For example, multichannel coherence (MC) measures can be employed to detect the amount of causal interaction between different brain regions, as shown in Duckrow and Spencer (1998). A more general approach is based on multichannel autoregressive models (MAR) (Kaminski and Blinowska 1991, Franaszczuk and Bergey 1999), or vector autoregressive models (VAR) (Kay 1988), which may be used to represent the entire pattern of interaction between several regions simultaneously. A successful application of the MAR model to depth electrode signals recorded from several parts of a rat's brain at rest and during motion is reported in Kaminski and Blinowska (1991). The approach is based on the estimation and interpretation of model parameters and reveals a significant increase of signal propagation between the cerebellum and the motor cortex during 
motion as compared to the rest stage. A similar technique was used in Franaszczuk and Bergey (1999) for the measurement of synchronization in ictal signals recorded by an array of electrodes implanted in the patient's brain (ICEEG). Increased synchrony was detected between the electrodes close to the epileptogenic focus during the preictal and ictal stages. This is in agreement with previous single channel studies showing a decrease of EEG signal complexity during these stages. This technique can be used to assess seizure spread patterns based on the depth electrode recordings of seizure onset.

Linear model-based methods for synchrony detection will generally work well only with electrophysiological data dominated by locally stationary signals (e.g., $\alpha$-rhythm, $\gamma$-rhythm, sustained ictal activity). The variance of a linear model parameter estimate is inversely proportional to the length of the data set, which makes it difficult to use these linear methods for the detection of interictal events propagation, since the events have a relatively brief duration, typically between 70 and $200 \mathrm{~ms}$. Also, surface conduction effects complicate linear modelbased methods applied to non-invasive signals. Constrained beamforming techniques only alleviate but do not solve the problem, due to non-zero spatial extent of the beam. Statistical significance testing should be used when linear methods are employed for synchrony and propagation detection in non-invasively obtained data (Nunez et al 1999).

An alternative promising approach to the analysis of depth electrode data is described in Bourien et al (2004). This method does not depend on linear measures, but instead uses data mining technique to search for statistically significant reproducible sequences of activations detected by a set of depth electrodes. The approach has been applied to detection of the propagation patterns associated with two different types of events: interictal spikes and transient bursts of fast activity. The technique is capable of finding physiologically plausible propagation networks based on the intracranial recordings. However, the specificity and sensitivity of event detection from non-invasively recorded data like EMEG are significantly lower than they are using intracranial depth electrode recordings. It would be desirable for propagation methods using extracranial data to incorporate non-ideal sensitivity and specificity explicitly.

Probabilistic models appear to offer an appropriate framework for coping with the inherent uncertainties in inferences from EMEG data. Probabilistic models have been used extensively for modelling the activity of neuronal assemblies on the micro level. Phenomenological spiking neuron models have a long tradition in theoretical neuroscience and are reviewed in Tuckwell (1988). Typically, probabilistic models of neuronal activity and propagation are concerned with simulation of chemical processes taking place inside a neuron. A model of spike propagation in neuronal networks with a small number of neurons was described in Kudela et al (1999). In Moradi (2004), Markov Models were used to simulate propagation of the neuronal excitation through a network of neurons with random excitatory connections. Markov Models were shown to adequately model propagation and survival of synchronous spiking in cortical neuronal networks. However, little work has been done on the application of such methods to the detection of neuronal activity propagation on the macroscopic level.

In the current work, we propose to use hidden Markov models (HMM) (Rabiner and Juang 1986) to infer propagation patterns between several potential epileptogenic regions, after the regions have been identified by automatic methods (Ossadtchi et al 2004) obtained from the analysis of interictal MEG data. As we will show, the HMM provides a framework for explicit modelling of propagation between the regions on the macroscopic level. HMMs also allow incorporation of specificity-sensitivity characteristics of the spike detection method by estimating state emission probability functions. We demonstrate that an analysis of the transition probability matrix allows us to draw conclusions regarding patterns of interictal activity propagation and to estimate physiological parameters of the interictal activity. 


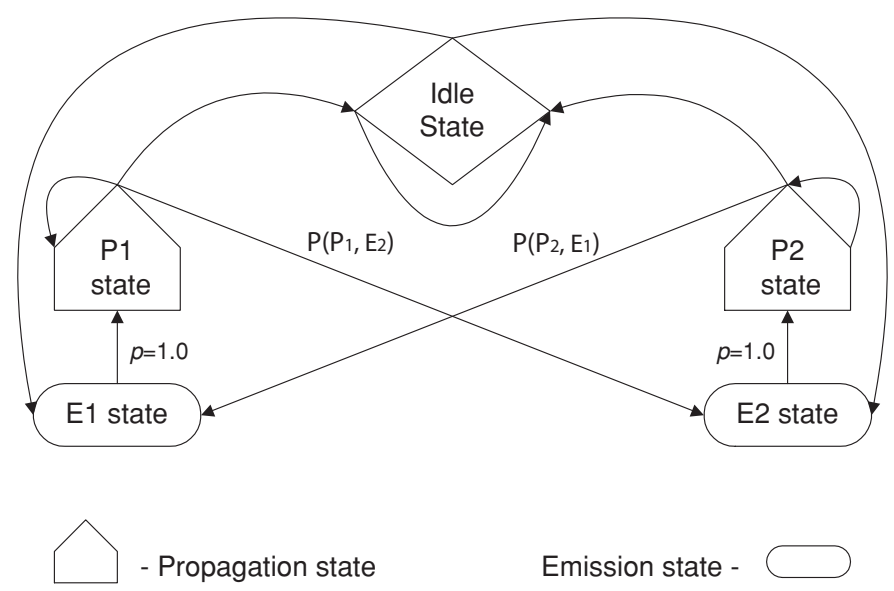

Figure 1. Simple two-cluster interaction Markov model. The number of foci is $N_{f}=2$, the number of states is $N_{s}=2 N_{f}+1=5$ and the number of allowed transitions is $N_{t}=11$.

In our presentation, we follow the inductive approach and first outline a two-focus model of interictal activity propagation based on the ideal spike detector assumption. We demonstrate that analysis of the transition probability matrix permits one to draw conclusions regarding patterns of interictal activity propagation and to estimate the physiological parameters of the propagation process. Next, we show how specificity-sensitivity characteristics of the detectors with discrete output can be incorporated into the model. The last step in the model description is its generalization for use with multiple foci.

Within the model identification section of the paper, we review the expectationmaximization (EM) and Baum-Welch algorithms used for identification of the transition probability matrix of our model. We then address issues of accuracy and robustness of the propagation detection procedure and report the results of both theoretical analysis and Monte Carlo simulations. We conclude with an example of the application of this method to a single patient. In a second paper, we will more thoroughly compare and interpret the results of applying the procedure to clinical MEG data collection from subjects with intractable multifocal epilepsy.

\section{Markov model for interictal activity propagation}

In the following, the propagation model describes the probability that an interictal spike directly results in the emission of another spike elsewhere. The observation model reflects the reality that we observe only a sequence of spikes and not the propagation state directly. We first describe a simple two-focus model and then generalize to an arbitrary number of foci.

\subsection{Propagation model}

We assume that time is indexed discretely as $t=0,1, \ldots, T-1$ at $f_{s}$ samples per second, such that the total passage of time $\tau$ is $\tau=T / f_{s}$ s or that correspondingly the total number of samples available is $T=\tau f_{s}$. Interictal activity as a function of time between $N_{f}=2$ foci is modelled by means of a Markov model shown in figure 1. At a time instance $t$ when a spike is detected in the $k$ th focus, the model is in the emission state $E_{k}$. In the next time instance $t+1$ the model transitions with probability 1 into a corresponding propagation state 
Table 1. Structure of the probability transition matrix $\mathbf{P}$ for the two-focus model. Asterisks indicate transitions corresponding to remaining in the same emitting state or to propagating from one focus to the other.

\begin{tabular}{llllll}
\hline & \multicolumn{5}{c}{$\mathcal{S}(t+1)$} \\
\cline { 2 - 6 } $\mathcal{S}(t)$ & $I$ & $P_{1}$ & $E_{1}$ & $P_{2}$ & $E_{2}$ \\
\hline$I$ & $p(I \mid I)$ & 0 & $p\left(E_{1} \mid I\right)$ & 0 & $p\left(E_{2} \mid I\right)$ \\
$P_{1}$ & $p\left(I \mid P_{1}\right)$ & $p\left(P_{1} \mid P_{1}\right)^{*}$ & 0 & 0 & $p\left(E_{2} \mid P_{1}\right)^{*}$ \\
$E_{1}$ & 0 & 1 & 0 & 0 & 0 \\
$P_{2}$ & $p\left(I \mid P_{2}\right)$ & 0 & $p\left(E_{1} \mid P_{2}\right)^{*}$ & $p\left(P_{2} \mid P_{2}\right)^{*}$ & 0 \\
$E_{2}$ & 0 & 0 & 0 & 1 & 0 \\
\hline
\end{tabular}

$P_{k}$. The model then remains in this propagation state for some elapsed time interval before either returning to an idle state $I$ or triggering the emission of a spike in the other focus. Our goal is to extract information about propagation between these pair of foci.

At every time instance, our model is therefore in one and only one of the following $N_{s}=2 N_{f}+1=5$ states:

- two emission states $\left(E_{1}\right.$ and $\left.E_{2}\right)$,

- two propagation states $\left(P_{1}\right.$ and $\left.P_{2}\right)$,

- one idle state $(I)$.

We denote the sequence of states as a function of time as $\mathcal{S} \equiv\{\mathcal{S}(t)\}$, where $\mathcal{S}(t)=s$, and $s \in\left\{I, P_{1}, P_{2}, E_{1}, E_{2}\right\}$. This model of propagation activity does not allow two spikes to occur simultaneously or at two consecutive time instances. For sufficiently high sampling rates of the analysed data, this property of the model is in agreement with the fact that $10-30 \mathrm{~ms}$ is required to generate an excitatory synaptic potential from the second population of neurons (Gloor 1985).

To further specify the Markov model, we need to introduce a probability transition matrix $\mathbf{P}$ with elements $\{p(B \mid A)\}$ defined as $p(B \mid A) \equiv \operatorname{Prob}(\mathcal{S}(t)=B \mid \mathcal{S}(t-1)=A)$, i.e. the conditional probability of being in state $B$ given we were in state $A$. Theoretically, a Markov model with five states would yield $N_{s}^{2}=25$ possible transitions, but physiological plausibility restricts the model to $N_{t}=11$ allowed transitions, indicated by the arrows in figure 1 . Absence of an arrow between a pair of states in a given direction indicates a banned state transition. Formally, restricted transitions are indicated by setting the corresponding elements of the transition probability matrix to 0 . The $\mathbf{P}$ matrix corresponding to the directed graph in figure 1 is shown in table 1 .

At time $t$, the probability of being in state $s, s \in\left\{I, P_{1}, P_{2}, E_{1}, E_{2}\right\}$, is denoted by the state probability vector $\pi(t)$, whose individual elements $\pi_{i}(t)$ represent the corresponding probability of being in that particular state. The probability in the next time instance is found using the state transition matrix, $\boldsymbol{\pi}(t+1)=\mathbf{P}^{T} \boldsymbol{\pi}(t)$. To initialize the Markov model, we need only specify the vector $\boldsymbol{\pi}(0)$ as the initial-state probability. The state probability vector at time $t$ is therefore $\boldsymbol{\pi}(t)=\left(\mathbf{P}^{T}\right)^{t} \boldsymbol{\pi}(0)$. Since spikes happen relatively rarely, and the segment of data that we analyse is more likely to start from the idle state than from an emission state, we may initially specify $\pi(0)=[1-4 \epsilon, \epsilon, \epsilon, \epsilon, \epsilon]^{T}, \epsilon \ll 1$.

A parameter of particular interest is the steady-state probability vector $\boldsymbol{\eta}$, such that $\mathbf{P}^{T} \boldsymbol{\eta}=\boldsymbol{\eta}$, which can be used for the analysis of a Markov model. We define $\eta_{i}$ as the element in $\boldsymbol{\eta}$ representing the steady-state transition probability for the $i$ th state in the set $s \in\left\{I, P_{1}, P_{2}, E_{1}, E_{2}\right\}$. The vector $\boldsymbol{\eta}$ can be computed as the appropriately normalized 
eigenvector corresponding to the unit eigenvalue of the transposed state transition probability matrix $\mathbf{P}^{T}$. For the two-focus case the required normalization condition is $\sum_{i=1}^{5} \eta_{i}=1$ such that the probabilities sum to unity.

With these definitions, the following physiological parameters, which describe interictal activity, can be computed as functions of the transition probability matrix:

- Elements $p\left(E_{2} \mid P_{1}\right)$ and $p\left(E_{1} \mid P_{2}\right)$ of the transition probability matrix reflect the probability at any instance that propagation from one focus will trigger an emission in the other focus.

- Given we are in the first propagation state $P_{1}$, the probability of propagation to the second emission state within time window $\left[\tau_{0}, \tau_{1}\right]$ seconds after entering the propagation state can be computed as

$$
\rho_{12}=p\left(E_{2} \mid P_{1}\right)\left(p\left(P_{1} \mid P_{1}\right)^{\tau_{0} f_{s}}-p\left(P_{1} \mid P_{1}\right)^{\tau_{1} f_{s}}\right) /\left(1-p\left(P_{1} \mid P_{1}\right)\right),
$$

and correspondingly for the reverse direction

$$
\rho_{21}=p\left(E_{1} \mid P_{2}\right)\left(p\left(P_{2} \mid P_{2}\right)^{\tau_{0} f_{s}}-p\left(P_{2} \mid P_{2}\right)^{\tau_{1} f_{s}}\right) /\left(1-p\left(P_{2} \mid P_{2}\right)\right) .
$$

- The expected time that either focus remains in its propagation state after emission can be computed as $\tau_{11}=\left(p\left(P_{1} \mid P_{1}\right) f_{s}\right)^{-1}$ and $\tau_{22}=\left(p\left(P_{2} \mid P_{2}\right) f_{s}\right)^{-1} \mathrm{~s}$.

- Over the entire observation of $T$ samples, the expected number of cases when a spike in the first focus is followed by a spike in the second focus can be computed as $Q_{12}=$ $\eta\left(P_{1}\right) p\left(E_{2} \mid P_{1}\right) T$. Correspondingly, for the reverse direction, $Q_{21}=\eta\left(P_{2}\right) p\left(E_{1} \mid P_{2}\right) T$.

\subsection{Observation model}

As discussed in section 1, each focus is assumed to be detected separately by a spike detection and source localization scheme, such as proposed in Ossadtchi et al (2004). For the two-focus model, the two detectors are modelled to have binary outputs $\mathcal{D}_{1}(t)$ and $\mathcal{D}_{2}(t)$, such that an active level of $\mathcal{D}_{k}(t)=1$ indicates emission of a spike from the $k$ th focus at time instance $t$; otherwise, $\mathcal{D}_{k}(t)=0$. For simplicity in the development, we encode the pair of binary outputs into single detector $\mathcal{D}(t)$ using a discrete representation scheme. According to this representation, the output of the pair of detectors is now represented by a scalar obtained as $\mathcal{D}(t)=2^{0} \mathcal{D}_{1}(t)+2^{1} \mathcal{D}_{2}(t)$. Thus, the scalar detector output is $\mathcal{D}(t)=d, d \in\{0,1,2,3\}$, and we can uniquely decode which detector indicated an emission.

Using this scheme, we observe that for a single observation $\mathcal{D}(t)$ we cannot uniquely distinguish the propagation and idle states of the five-state Markov model in figure 1, since both states yield $\mathcal{D}_{k}(t)=0$. The only way to distinguish these states is by an analysis of the entire set of observations, $\mathcal{D} \equiv\{\mathcal{D}(t)\}$, discussed in the next section. We further note that the detectors $\mathcal{D}_{k}$ are imperfect, with specificity values $v_{k}$ and sensitivity values $\mu_{k}, k \in\{1,2\}$, defined as

$$
\begin{aligned}
& v_{k}=p\left(\mathcal{D}_{k}(t)=0 \mid \text { no spike at time } t \text { in the } k \text { th focus }\right), \\
& \mu_{k}=p\left(\mathcal{D}_{k}(t)=1 \mid \text { a spike at time } t \text { in the } k \text { th focus }\right) .
\end{aligned}
$$

In other words, the detectors have a 'false alarm rate' (FAR) of $\left(1-v_{k}\right)$ and a 'probability of missed detection' of $\left(1-\mu_{k}\right)$. Thus, for example, our pair of detectors may simultaneously declare an emission at time $t$, such that $\mathcal{D}(t)=3$, where one or both of the detectors may have triggered a 'false alarm,' even though our state model precludes both foci simultaneously emitting.

We therefore model the probability of the detector output using a state emission probability mass function $\psi_{s}(d) \equiv \operatorname{Prob}(d=\mathcal{D}(t) \mid s=\mathcal{S}(t))$, i.e. the probability of observing the 


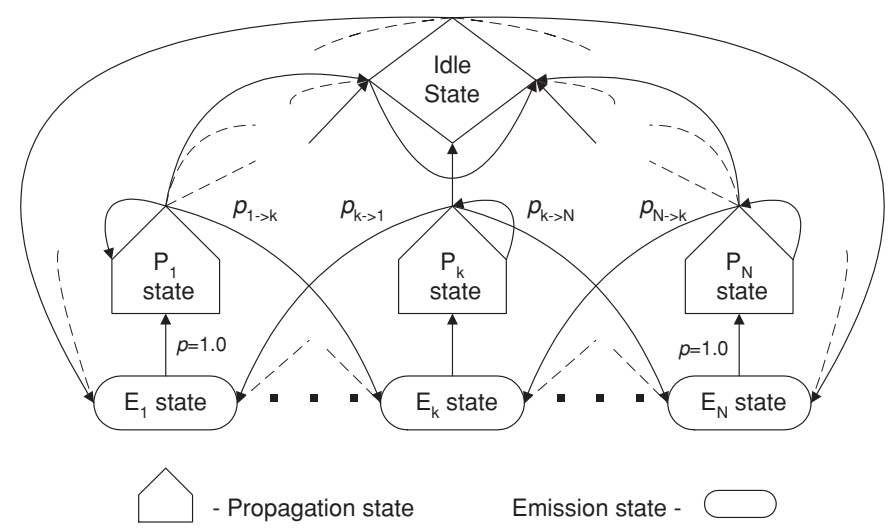

Figure 2. Simple $N$-cluster interaction Markov model. The number of foci is $N_{f}$, the number of states is $N_{s}=2 N_{f}+1$ and the number of allowed transitions is $N_{t}=4 N_{f}+2\left(N_{f} !\right) /$ $\left(2 !\left(N_{f}-2\right) !\right)+1$.

Table 2. For the two-focus model, the probability of detector outputs $d \in\{0,1,2,3\}$, given the states $s \in\left\{I, P_{1}, P_{2}, E_{1}, E_{2}\right\}$. Each detector $k \in 1,2$ has specificity $v_{k}$ and sensitivity $\mu_{k}$ for detecting an emission $E_{k}$. The scalar detector output $d$ is the encoded sum of the individual binary detector outputs, $d=d_{1}+2 d_{2}$.

\begin{tabular}{lllll}
\hline \multicolumn{4}{c}{ Detector output $d$} \\
\hline State $s$ & $\psi_{s}(0)$ & $\psi_{s}(1)$ & $\psi_{s}(2)$ & $\psi_{s}(3)$ \\
\hline$I, P_{1}, P_{2}$ & $v_{1} v_{2}$ & $\left(1-v_{1}\right) v_{2}$ & $v_{1}\left(1-v_{2}\right)$ & $\left(1-v_{1}\right)\left(1-v_{2}\right)$ \\
$E_{1}$ & $\left(1-\mu_{1}\right) v_{2}$ & $\mu_{1} v_{2}$ & $\left(1-\mu_{1}\right)\left(1-v_{2}\right)$ & $\mu_{1}\left(1-v_{2}\right)$ \\
$E_{2}$ & $v_{1}\left(1-\mu_{2}\right)$ & $\left(1-v_{1}\right)\left(1-\mu_{2}\right)$ & $v_{1} \mu_{2}$ & $\left(1-v_{1}\right) \mu_{2}$ \\
\hline
\end{tabular}

detector output $d \in\{0,1,2,3\}$ given we are in state $s \in\left\{I, P_{1}, P_{2}, E_{1}, E_{2}\right\}$. The state emission probability mass functions $\psi_{s}(d)$ allow us to take into account realistic specificity $v_{k}$ and sensitivity $\mu_{k}$ characteristics of the discrete detectors $\mathcal{D}_{k}, k \in\{1,2\}$, as shown in table 2 .

\subsection{Generalization to multiple foci}

The approach for two foci can now be generalized to model activity propagation between an arbitrary number of foci. In general, in order to model propagation between $N_{f}$ foci we need to use a structure shown in figure 2 with $N_{s}=2 N_{f}+1$ hidden states and $N_{t}=4 N_{f}+2 C_{2}^{N_{f}}+1$ allowed transitions, where $C_{n}^{N}$ stands for number of combinations of $N$ items taken $n$ at a time, $C_{n}^{N} \equiv N ! /(n !(N-n) !)$. Only one emission $E_{k}$ can occur in any time instance, where it then transitions in the next time instance with probability 1 to its propagation state $P_{k}$. From the propagation state, the focus either eventually transitions to the idle state $I$ or transitions directly to a new emission state.

The state sequence is again denoted as a function of time, $\mathcal{S}(t)=s$, where now $s \in\left\{I, P_{1}, \ldots, P_{N_{f}}, E_{1}, \ldots, E_{N_{f}}\right\}$. The initial-state probability vector may again be specified as $\boldsymbol{\pi}(0)=\left[1-2 \epsilon N_{f}, \epsilon, \ldots, \epsilon\right], \epsilon \ll 1$. The probability transition matrix $\mathbf{P}$ is the obvious multiple-focus extension of the two-focus case, where many probabilities are set to 0 in order 
to prohibit transitions. Vector $\boldsymbol{\eta}$, as in the two-focus case, is computed as the appropriately normalized eigenvector corresponding to the unit eigenvalue of the transposed state transition probability matrix $\mathbf{P}^{T}$. The normalization condition is $\sum_{i=1}^{2 N_{f}+1} \eta_{i}=1$.

Based on the probability transition matrix, the following set of physiological propagation parameters can be computed:

- Elements $p\left(E_{l} \mid P_{k}\right)$ of the transition probability matrix reflect the probability at any instance that propagation in focus $k$ will trigger an emission in focus $l$.

- Given we are in propagation state $P_{k}$, the probability of propagation to the emission state $E_{l}$ within time window $\left[\tau_{0}, \tau_{1}\right]$ seconds after entering the propagation state can be computed as

$$
\rho_{k l}=p\left(E_{l} \mid P_{k}\right)\left(p\left(P_{k} \mid P_{k}\right)^{\tau_{0} f_{s}}-p\left(P_{k} \mid P_{k}\right)^{\tau_{1} f_{s}}\right) /\left(1-p\left(P_{k} \mid P_{k}\right)\right) .
$$

- The expected time that any focus remains in its propagation state $P_{k}$ after emission can be computed as $\tau_{k k}=\left(p\left(P_{k} \mid P_{k}\right) f_{s}\right)^{-1} \mathrm{~s}$.

- Over the entire observation of $T$ samples, the expected number of cases when a spike in the $k$ th focus is followed by a spike in the lth focus can be computed as $Q_{k l}=\eta\left(P_{k}\right) p\left(E_{l} \mid P_{k}\right) T$.

The propagation model for $N_{f}$ foci is supported by a set of $N_{f}$ detectors $\mathcal{D}_{k}$ with binary outputs $\mathcal{D}_{k}(t)=d_{k}, d_{k} \in\{0,1\}, k=1, \ldots, N_{f}$. The scalar observable output $\mathcal{D}(t)$ is encoded as $\mathcal{D}(t)=\mathcal{D}_{1}(t)+2 \mathcal{D}_{2}(t)+\cdots+2^{\left(N_{f}-1\right)} \mathcal{D}_{N_{f}}(t)$, which takes on the values from the set $\mathcal{D}(t)=d, d \in\left\{0, \ldots, 2^{N_{f}}-1\right\}$. In order to generalize an expression for the state emission probability density function $\psi_{s}(d)$, we extend our concept of the binary encoding of $d$ by converting it to a bit sequence comprising $N_{f}$ bits. We represent the binary encoding of the output $d$ using binary vector $\mathbf{b}^{d}$, such that $d=\sum_{k=1}^{N_{f}} b_{k}^{d} 2^{(k-1)}$; in other words, the $k$ th bit in $\mathbf{b}^{d}$ is represented by detector $\mathcal{D}_{k}(t)$. Using this encoding, we can generalize the expression for $\psi_{s}(d)$ in the idle or propagation state as

$$
\psi_{s}(d)=\prod_{k=1}^{N_{f}} v_{k}^{\overline{b_{k}^{d}}}\left(1-v_{k}\right)^{b_{k}^{d}}, \quad s \in\left\{I, P_{1}, \ldots, P_{N_{f}}\right\}
$$

where $\overline{b_{k}^{d}}$ denotes the logical negation of the $k$ th bit.

We next generalize the expression for $\psi_{s}(d)$ for the emission states $s=\left\{E_{1}, \ldots, E_{N_{f}}\right\}$. For convenience, we introduce another binary vector variable $\mathbf{b}^{k}$, where $k$ corresponds to emission state $E_{k}$. The number of bits in this vector is equal to that in the vector $\mathbf{b}^{d}$, but the encoding scheme is positional, i.e. all bits except for the $k$ th bit of vector $\mathbf{b}^{k}$ are 0 and the $k$ th bit is set to 1 . Now let us introduce another vector of bits $\Delta$ for which each bit is computed as an XOR (exclusive OR) of the corresponding bits of $\mathbf{b}^{k}$ and $\mathbf{b}^{d}$. In other words, $\Delta_{l}^{d k}=b_{l}^{d} \oplus b_{l}^{k}$. Then the expression for the $\psi_{s}(d)$ for $s=\left\{E_{1}, \ldots, E_{N_{f}}\right\}$ can be written as

$$
\psi_{s}(d)=\prod_{l=1}^{N_{f}}\left[\mu_{l}^{\overline{\Delta_{l}^{d k}}}\left(1-\mu_{l}\right)^{\Delta_{l}^{d k}}\right]^{\delta_{l k}}\left[v_{l}^{\overline{\Delta_{l}^{d k}}}\left(1-v_{l}\right)^{\Delta_{l}^{d k}}\right]^{\overline{\delta_{l k}}} s \in\left\{E_{k}\right\}
$$

where $\delta_{l k}$ is a Kronecker symbol and the overbar again denotes logical negation.

In the above, $v_{l}$ and $\mu_{l}$ are correspondingly the specificity and sensitivity of the $l$ th detector. The formulae given above allow the construction of the HMM for the propagation detection between an arbitrary number of foci. 


\section{Parameter estimation}

We assume that we have the sequence of observed detector outputs $\mathcal{D}=\{\mathcal{D}(t)\}, t=1, \ldots, T$ from which we wish to infer the state sequence $\mathcal{S}=\{\mathcal{S}(t)\}$ and the probability transition matrix $\mathbf{P}$ of the HMM. In order to estimate $\mathbf{P}$, we discuss below the use of the expectationmaximization (EM) algorithm. We then examine the error variance, interpretation, significance and applicability of generating these estimates.

\subsection{Constrained EM algorithm}

We use the EM algorithm as described in (Rabiner and Juang 1993) to estimate the parameters of our HMM. Each iteration of the algorithm is comprised of two steps. During the $n$th iteration within the expectation $(E)$ step, the algorithm computes the auxiliary function $\mathcal{Q}\left(\Theta_{n}, \Theta_{n-1}\right)$ equal to the expectation of the likelihood function $\mathcal{L}\left(\Theta_{n} \mid \mathcal{D}, \mathcal{S}\right)$ over all possible values of the hidden data $\mathcal{S}$, for the observed data $\mathcal{D}$ and a set of parameters $\Theta_{n-1}$ obtained during the maximization step of the $(n-1)$ th iteration of the algorithm. The maximization $(M)$ step computes new values for the set of parameters $\Theta_{n}$ which maximize the auxiliary function $\mathcal{Q}\left(\Theta_{n}, \Theta_{n-1}\right)$. In our application, we define the parameter set to be the state transition matrix and the initial-state probabilities, $\Theta$ as $\Theta=\{\mathbf{P}, \boldsymbol{\pi}(0)\}$.

For notational convenience, we now introduce the equivalent notation $\mathcal{S}(t)=i$ to indicate the $i$ th element of the possible state set $\left\{I, P_{1}, \ldots, P_{N_{f}}, E_{1}, \ldots, E_{N_{f}}\right\}$. We specify the state emission function $\psi_{s}(d)$ for the $i$ th state as $\psi_{i}(d)$, based on our prior knowledge of the characteristics of the detectors $\mathcal{D}_{k}, k=1, \ldots, N_{f}$, according to equations (2) and (3). As mentioned previously, we initialize the state probability vector as $\pi^{0}=\left[1-2 \epsilon N_{f}, \epsilon, \ldots, \epsilon\right]$, and $\pi_{i}^{n}$ is the probability for the $i$ th state at the $n$th iteration. Finally, we initialize the transition probability matrix $\mathbf{P}^{0}$ with $p_{i j}^{0}=0$ for those transitions that are not allowed, $p_{i j}^{0}=1$ for the mandatory transitions and $p_{i j}^{0}=\epsilon_{i}$ for the rest of the transitions, where $\epsilon_{i}$ is selected in such a way that $\sum_{j} p_{i j}^{0}=1$. In the above, we used the compact notation $p_{i j}^{n} \equiv \operatorname{Prob}(j=\mathcal{S}(t+1) \mid i=\mathcal{S}(t))$ at the $n$th iteration.

At the $n$th iteration of the EM algorithm the following probabilities are introduced to facilitate computation of the auxiliary function $\mathcal{Q}$ :

- $\alpha_{j}^{n}(t)=\operatorname{Prob}\left(d=\mathcal{D}(1), \ldots, d=\mathcal{D}(t), j=\mathcal{S}(t) \mid \Theta_{n-1}\right)$,

- $\beta_{i}^{n}(t)=\operatorname{Prob}\left(d=\mathcal{D}(t+1), \ldots, d=\mathcal{D}(T-1), i=\mathcal{S}(t) \mid \Theta_{n-1}\right)$.

In other words, at the $n$th iteration of the EM algorithm $\alpha_{j}^{n}(t)$ represents the joint probability of observing the current sequence of detector outputs up to time $t$ and the $j$ th state in $s \in\left\{I, P_{1}, \ldots, P_{N_{f}}, E_{1}, \ldots, E_{N_{f}}\right\}$ at time $t$, given the estimate in the previous $(n-1)$ th iteration for the parameters in $\Theta$. We refer to $\alpha_{j}^{n}(t)$ as a forward joint probability. Similarly, $\beta_{i}^{n}(t)$ is the backward joint probability of all of the future observed detector outputs and the current state.

At the $n$th iteration, these parameters are initialized as

$$
\begin{aligned}
& \alpha_{j}^{n}(0)=\pi_{j}^{n} \psi_{j}(\mathcal{D}(0)) \\
& \beta_{i}^{n}(T-1)=1 .
\end{aligned}
$$

We then use the Baum-Welch algorithm to recursively compute the values of $\alpha_{j}^{n}(t)$ and $\beta_{i}^{n}(t)$ according to the following expressions:

$$
\alpha_{j}^{n}(t+1)=\psi_{j}(\mathcal{D}(t+1)) \sum_{i=1}^{N_{s}} \alpha_{i}^{n}(t) p_{i j}^{n} \quad t=0, \ldots, T-2
$$




$$
\beta_{i}^{n}(t)=\sum_{j=1}^{N_{s}} \beta_{j}^{n}(t+1) p_{i j}^{n} \psi_{j}(\mathcal{D}(t+1)) \quad t=T-2, \ldots, 0 .
$$

Using the backward and forward probabilities the following additional probabilities are introduced:

- $\xi_{i j}^{n}(t)=\operatorname{Prob}\left(i=\mathcal{S}(t), j=\mathcal{S}(t+1) \mid \mathcal{D}, \Theta_{n-1}\right)$,

- $\gamma_{i}^{n}(t)=\operatorname{Prob}\left(i=\mathcal{S}(t) \mid \mathcal{D}, \Theta_{n-1}\right)$,

which at the $n$th iteration are computed using the forward and backward probabilities as

$$
\xi_{i j}^{n}(t)=\frac{\alpha_{i}^{n}(t) p_{i j}^{n} \psi_{j}(\mathcal{D}(t+1)) \beta_{j}^{n}(t+1)}{\sum_{i=1}^{N_{s}} \sum_{j=1}^{N_{s}} \alpha_{i}^{n}(t) p_{i j}^{n} \psi_{j}(\mathcal{D}(t+1)) \beta_{j}^{n}(t+1)}
$$

and

$$
\gamma_{i}^{n}(t)=\frac{\alpha_{i}^{n}(t) \beta_{i}^{n}(t)}{\sum_{i=1}^{N_{s}} \alpha_{i}^{n}(t) \beta_{i}^{n}(t)} .
$$

Parameter updates are then computed according to the following pair of equations:

$$
\begin{aligned}
\pi_{i}^{n+1} & =\gamma_{i}^{n}(1) \\
p_{i j}^{n+1} & =\frac{\sum_{t=0}^{T-2} \xi_{i j}^{n}(t)}{\sum_{t=0}^{T-1} \gamma_{i}^{n}(t)} .
\end{aligned}
$$

The recursion is therefore defined by equations (4)-(11). The probability transition matrix at the $n$th iteration is formed from the individual probabilities, $\mathbf{P}^{n} \equiv\left[p_{i j}^{n}\right]$. We terminate the recursion according to the following criterion:

$$
\left\|\mathbf{P}^{n}-\mathbf{P}^{n-1}\right\|_{F}<\epsilon N_{s}^{2}
$$

where \|\|$_{F}$ denotes the Frobenius norm of the matrix and $\epsilon$ is a small number. Our final estimates are the probability transition matrix $\hat{\mathbf{P}}=\mathbf{P}^{n}$ and the initial-state probability vector $\hat{\pi}(0)=\pi^{n}$.

\subsection{Theoretical bounds}

We now calculate the variance of the estimates of the transition probability matrix $\mathbf{P}$. The classical way to derive the lower bound for the variance of an unbiased estimate of a parameter would be to derive the Cramer-Rao lower bound based on the log-likelihood function. The parameters in the rows of the state transition matrix must sum to unity so that they cannot be treated as independent variables. This makes direct calculation of the Cramer-Rao bound rather difficult. An alternative expression for the lower bound can be derived by first finding a minimum variance unbiased (MVU) estimator for the $p(j \mid i)$ 's and then computing their variance (Kay 1993). Here, we will continue the previous section's indexing scheme for states $s \in\left\{I, P_{1}, \ldots, P_{N_{f}}, E_{1}, \ldots, E_{N_{f}}\right\}$. A MVU estimator for the elements of the transition probability matrix exists and is obtained by simply counting the transition events from state $i$ to state $j$ and computing the ratio to the total number of times the system was in state $i$, i.e.

$$
\hat{p}(j \mid i)=\frac{N(j \mid i)}{N(i)},
$$

where $N(i)$ is the number of events observed in the $i$ th state and $N(j \mid i)$ is the number of transitions observed from the $i$ th state to the $j$ th state. In appendix A.1, using the notion of sufficient statistics we prove that the estimator (13) is minimum variance and unbiased. 


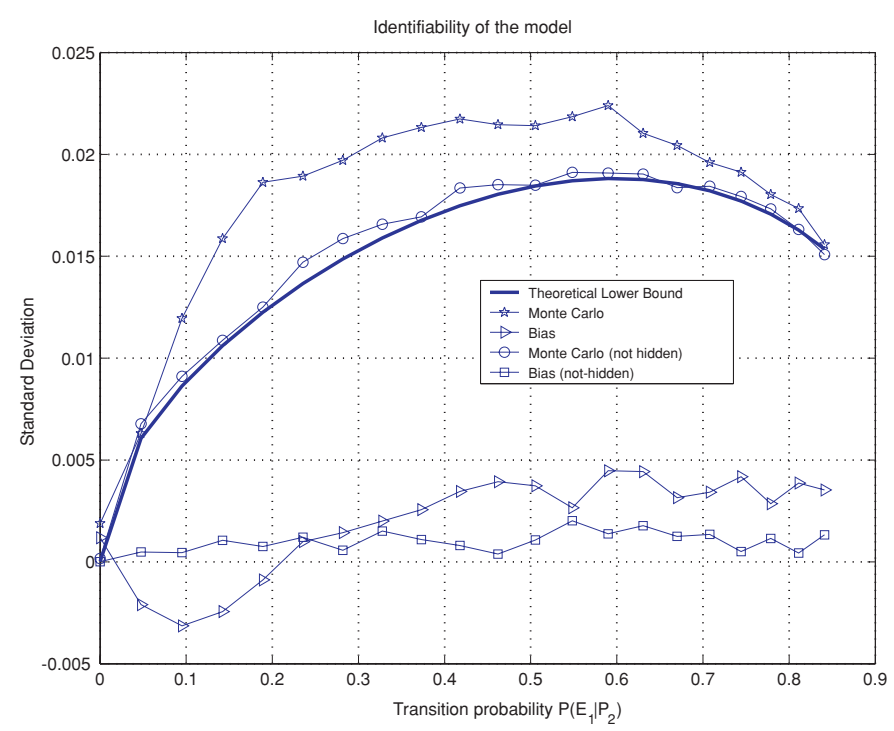

Figure 3. Comparison of variances of estimates of $p\left(E_{1} \mid P_{2}\right)$. The Monte Carlo simulation estimate of the Markov chain (o) using the ratio estimator confirms the theoretical bound (solid line) on the variance for the non-hidden Markov model, i.e. when the states can be observed directly. The plot also shows the bias of this estimate ( $\square$ ). Also shown are the variance $(*)$ and the bias $(\triangleright)$ of the EM estimate for the hidden Markov model.

The minimum variance estimator (13) achieves the lower bound on the variance of the estimate. In appendix A.2, we show that the variance of the estimator $\hat{p}(j \mid i)=N(j \mid i) / N(i)$ can be computed as

$$
\operatorname{var}\left\{\frac{N(j \mid i)}{N(i)}\right\}=p(j \mid i)(1-p(j \mid i))\left(\frac{1}{T \eta_{i}}+\frac{1-\eta_{i}}{T^{2} \eta_{i}^{2}}\right)+o\left(\frac{1}{\eta_{i} T^{3}}\right),
$$

where $T$ is the length of the data set and $\boldsymbol{\eta}$ is the steady-state probability vector.

To compare the standard deviation of the transition probability estimate $\hat{p}\left(E 1 \mid P_{2}\right)_{\mathrm{EM}}$ obtained by the EM algorithm in the hidden case with the theoretical lower bound (14), we performed a set of Monte Carlo (MC) simulations. We generated sequences using the Markov chain structure shown in figure 1 with state emission probability mass functions specified in table 2. We performed simulations for different values of the transition probability $p\left(E 1 \mid P_{2}\right)$ in the interval $[0,0.85]$ and for the data set length $T=5000$. The results of these simulations are shown in figure 3.

In order to verify the accuracy of the bound in (14), we also show the standard deviation of the 'ratio' estimate $\hat{p}\left(E_{1} \mid P_{2}\right)_{\mathrm{RAT}}=N\left(E_{1} \mid P_{2}\right) / N\left(P_{2}\right)$ computed from the Monte Carlo simulated data. This estimate requires that we know the state of the model at each point in time, which in practice we do not since the propagation and idle states produce identical observations. The bound coincides well with the standard deviation of the Monte Carlo ratio estimate. In the same figure, we also plot the biases of the estimates of $p\left(E_{1} \mid P_{2}\right)$ for the ratio estimate in the non-hidden case and the EM estimate for the hidden case.

The Monte Carlo simulations show that the standard deviation of the estimate $\hat{p}\left(E_{1} \mid P_{2}\right)_{\mathrm{EM}}$ is at most 1.4 times larger than the smallest possible standard deviation delivered by the $\hat{p}\left(E_{1} \mid P_{2}\right)_{\mathrm{RAT}}=N\left(E_{1} \mid P_{2}\right) / N\left(P_{2}\right)$ estimator. Also, in reality we expect the propagation time to be greater than $20 \mathrm{~ms}$ resulting in transition probability values less than 0.2 for the sample 


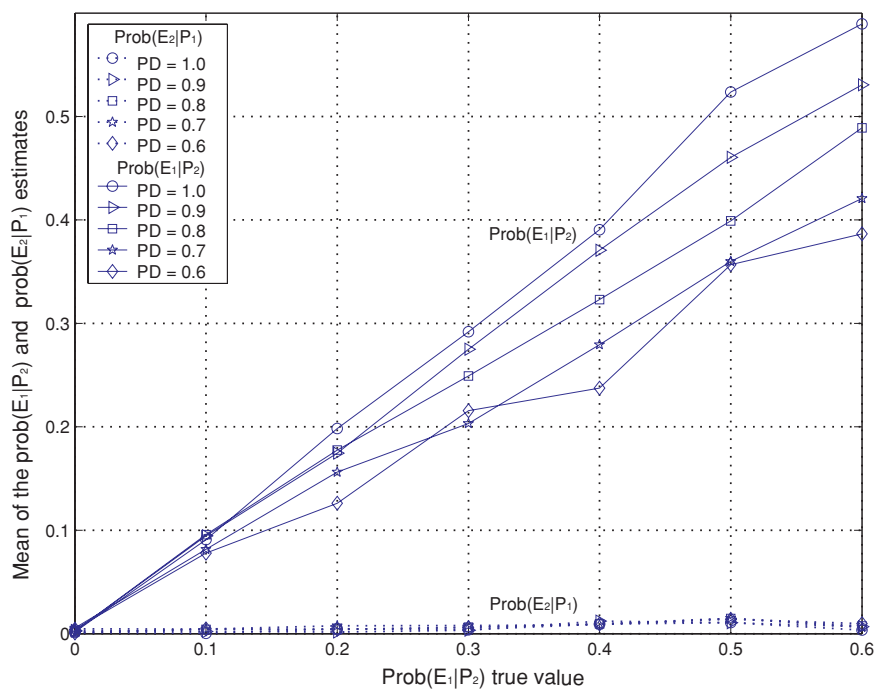

(a)

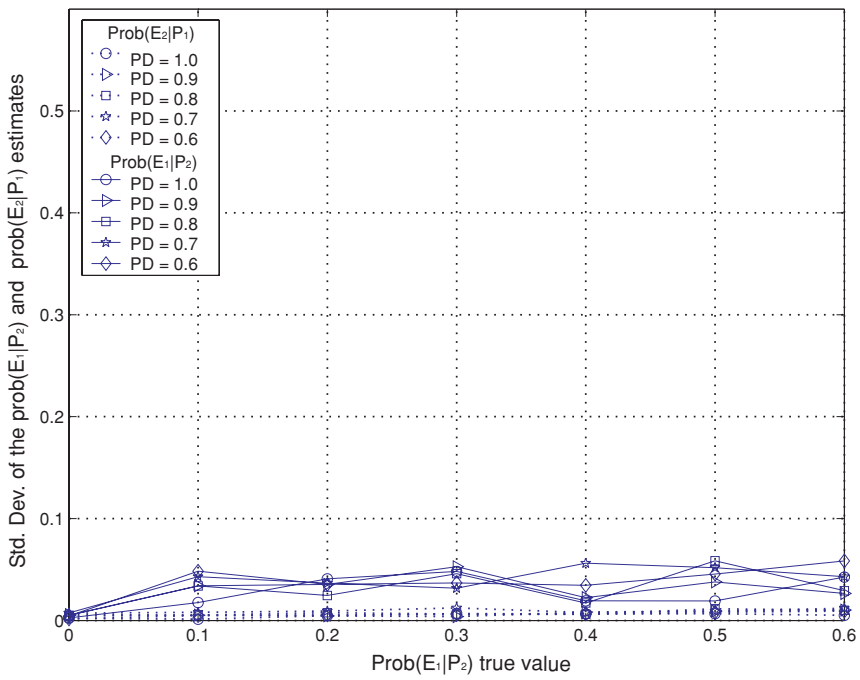

(b)

Figure 4. Effects of imperfect detection (PD) on the mean (a) and the standard deviation (b) of the transition probability estimate, if we instead assume perfect detection. (c) and (d) show that incorporating the imperfect detector characteristics $\psi_{s}(d)$ into the observation model allows us to obtain approximately unbiased estimates of the transition probability matrix (c), and the standard deviation (d) shows only a moderate increase.

rate of 250 samples s ${ }^{-1}$. This allows us to conclude that the EM estimator can be successfully used for transition probability estimation in the hidden framework.

\subsection{Imperfect specificity-sensitivity}

In real applications, the detectors $\mathcal{D}_{i}, i=1, \ldots, N_{f}$, are imperfect, and we have accounted for realistic specificity-sensitivity characteristics using the probability mass function $\psi_{s}(d)$ shown in table 2. In this section, we explore the consequences of ignoring realistic detection 


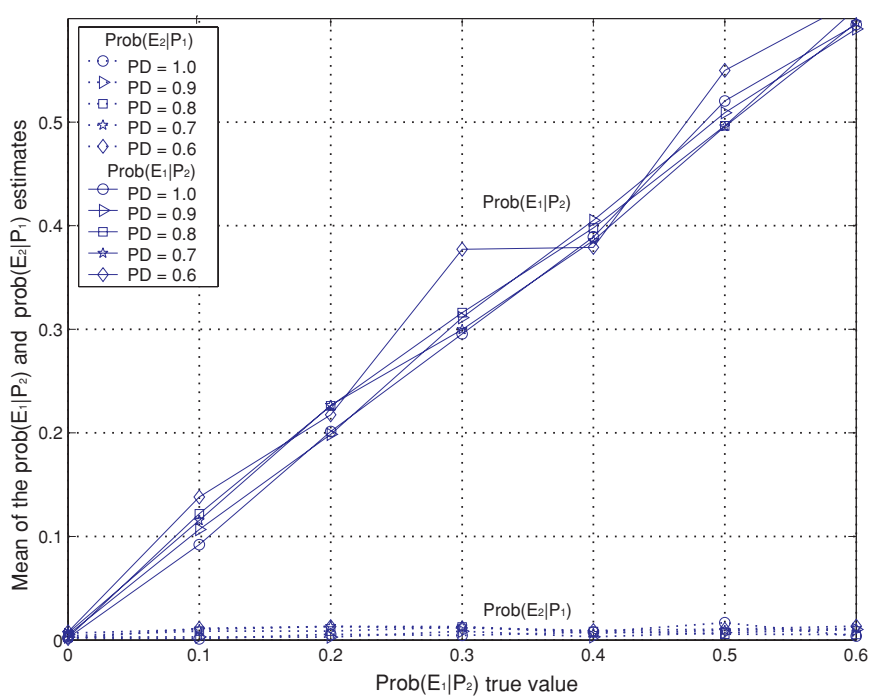

(c)

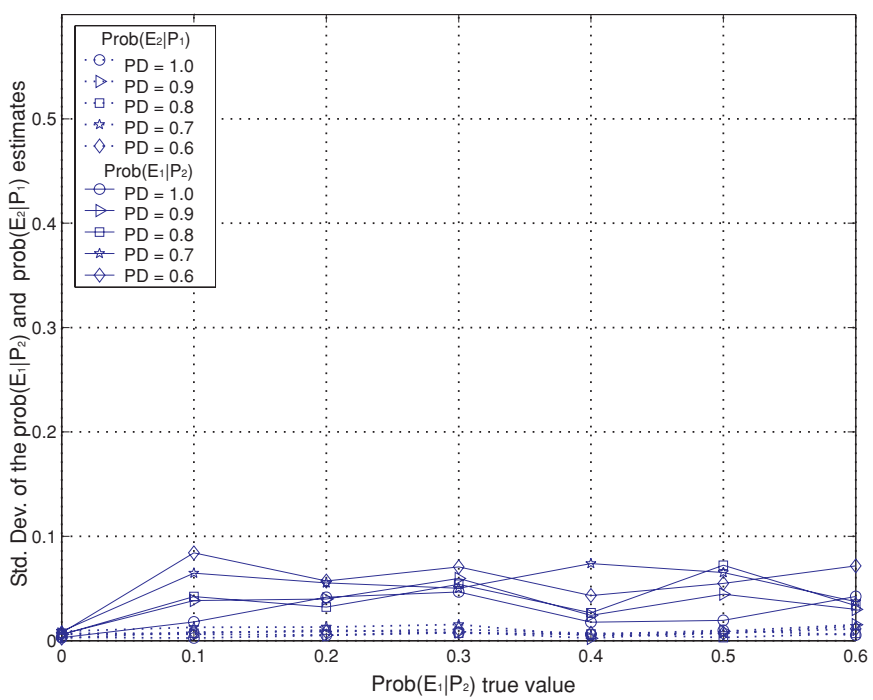

(d)

Figure 4. (Continued.)

probabilities and simply assuming perfect detection. We show that if we assume perfect detection, then the actual imperfect specificity-sensitivity characteristics of detectors will result in increased variance and bias of the estimates.

We report the results of a Monte Carlo study using the HMM structure in figure 1 for both the case of incorrectly assuming perfect detection and the case of correctly including imperfect characteristics. In this two-focus simulation, we set the probability of propagation from the first focus to the second focus to be identically $0, p\left(E_{2} \mid P_{1}\right)=0$ and vary the propagation in the other direction from $p\left(E_{1} \mid P_{2}\right)=0$ to $p\left(E_{1} \mid P_{2}\right)=0.6$. For each probability of propagation, we vary either the probability of detection (PD, or sensitivity) from $60 \%$ to perfect, or the 


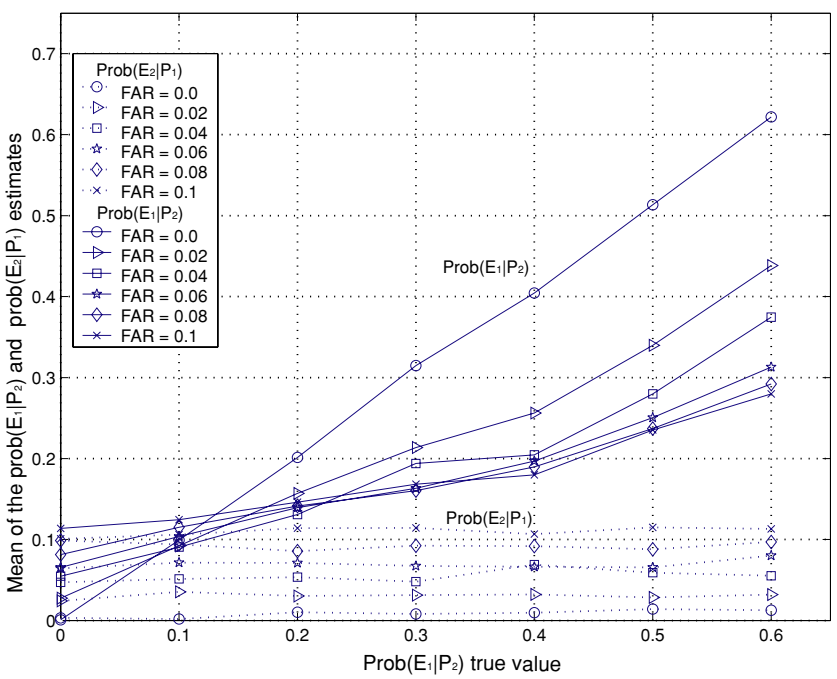

(a)

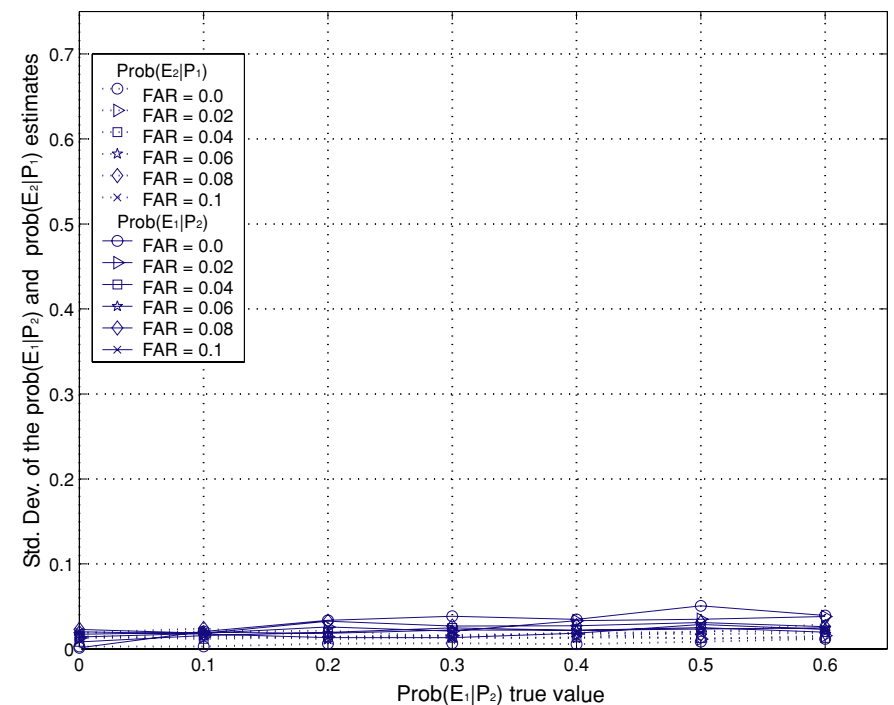

(b)

Figure 5. (a) Effects of non-ideal specificity (non-zero false alarm rate (FAR)) on the mean (a) and the standard deviation (b) of the transition probability estimate for different specificity values, where FAR $=1-$ specificity. (c) and (d) show the effect of including the actual specificity of the detectors $\psi_{s}(d)$ in the observation model on (c) bias and (d) standard deviation.

probability of false alarm ( 1 - specificity) from perfect (none) to $10 \%$. Twenty Monte Carlo trials were performed for each combination of parameters $p\left(E_{1} \mid P_{2}\right), p\left(E_{2} \mid P_{1}\right), v$ and $\mu$.

We first examine the impact of imperfect detection (PD $<1)$. Figures $4(a)$ and (b) shows the effects of assuming perfect detection in the presence of imperfect sensitivity of the detectors. We can conclude that as detector sensitivity decreases, the mean of $p\left(E_{1} \mid P_{2}\right)$ decreases and the estimate acquires a multiplicative bias. The mean of the estimate of $p\left(E_{2} \mid P_{1}\right)$ remains correctly very close to 0 and is unaffected by the imperfect sensitivity of 


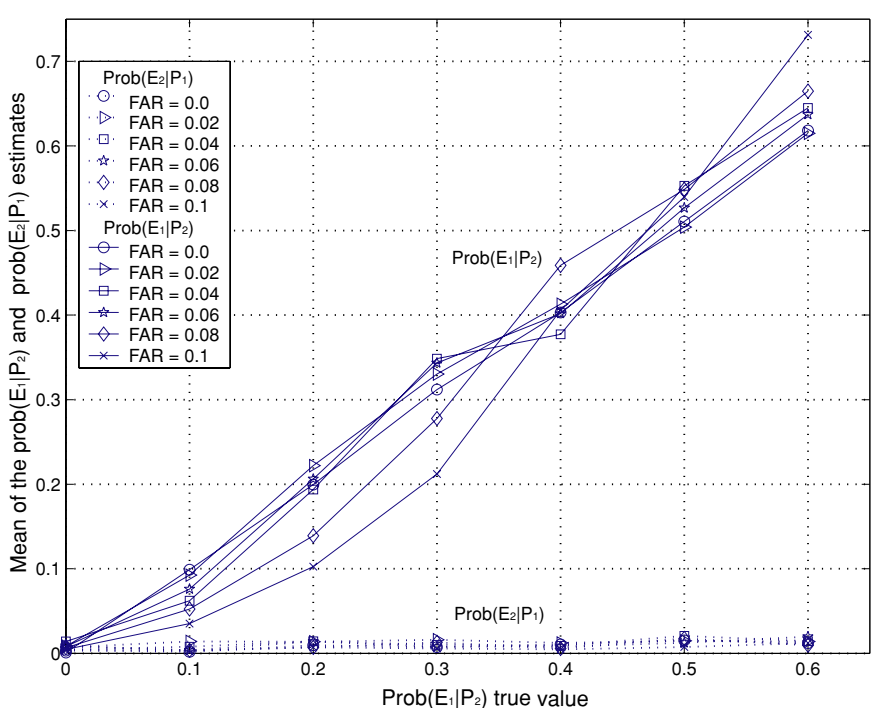

(c)

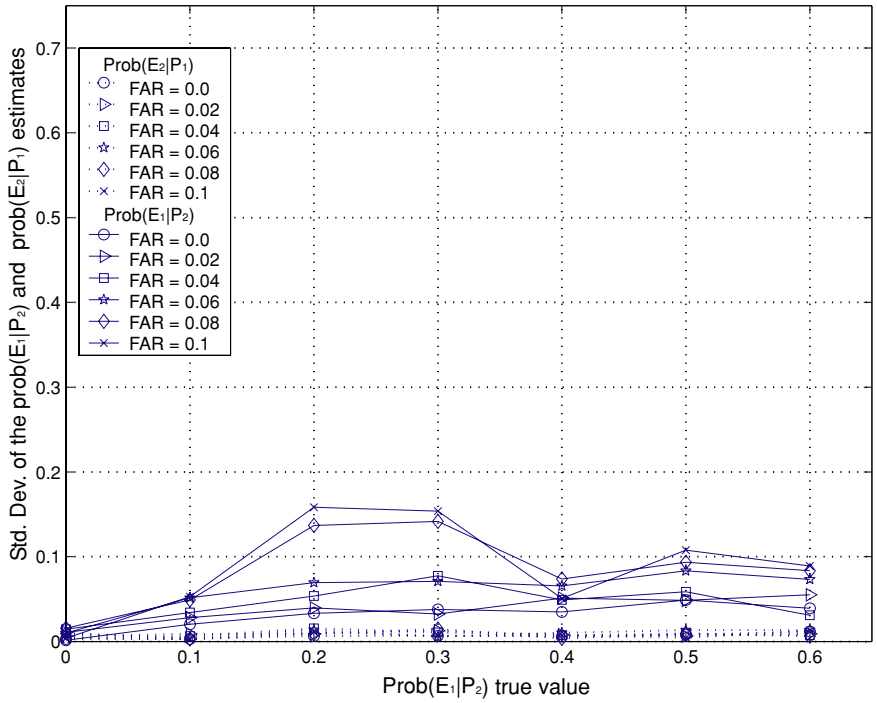

(d)

Figure 5. (Continued.)

the detector. As sensitivity decreases, the contrast between estimates of zero and non-zero transition probability values still remains detectable even for very low $(\mathrm{PD}=0.6)$ values of detector sensitivity. The standard deviation of the estimate is unaffected by the non-ideal sensitivity and remains constant for all positive values of the transition probability.

The effects of including the information on non-ideal sensitivity into the algorithm are shown in figures 4(c) and (d). One can see that the multiplicative bias disappears and the mean of the estimate $\hat{p}\left(E_{1} \mid P_{2}\right)$ is very close to the true value of $p\left(E_{1} \mid P_{2}\right)$. The cost is a moderate increase in the standard deviations of the estimate of $p\left(E_{1} \mid P_{2}\right)$. 

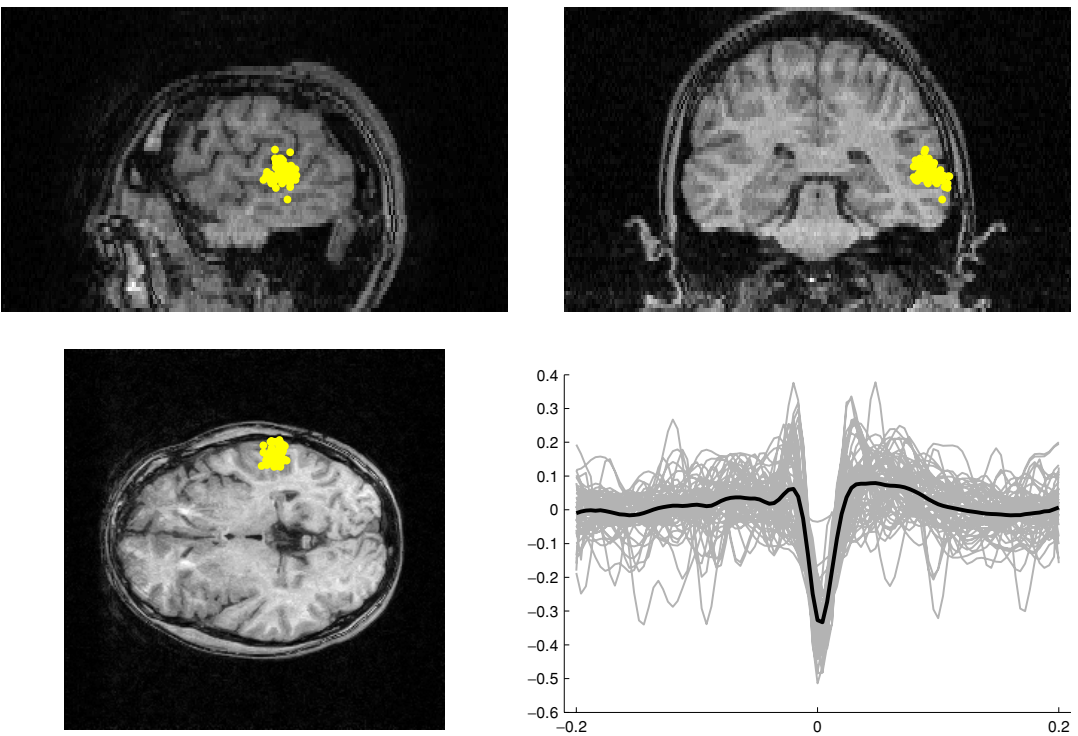

(a)
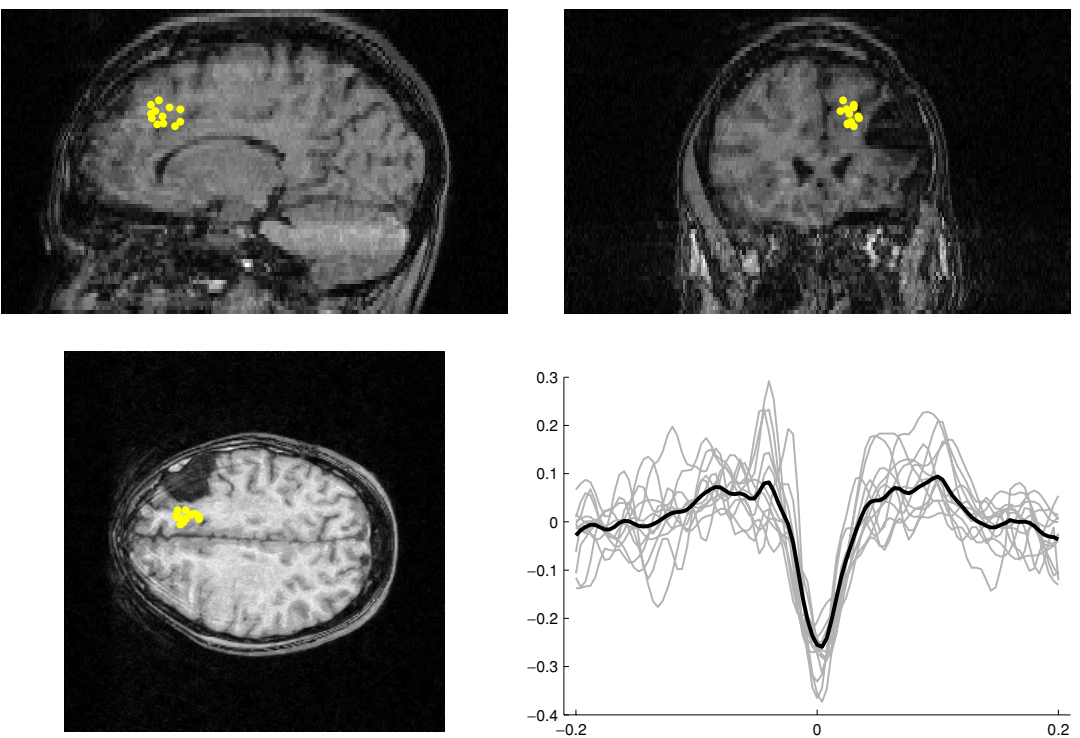

(b)

Figure 6. Three statistically significant clusters of dipoles detected in the first data set: (a) cluster 1, (b) cluster 2 and (c) cluster 3 . The other three data sets produced comparable results. Also displayed are overlays and the average spike shape for each cluster.

We next examine the impact of imperfect specificity, i.e. the 'false alarm rate' (FAR) is non-zero. The specificity of $v$ corresponds to a FAR of $1-v$ and results in $(1-v)\left(T-N_{s}\right)$ false detections, where $T$ is the data set duration (in samples) and $N_{s}$ is the number of true spikes. Thus, for $N_{s}=100$ and a total data set duration of $T=4000$ samples with a specificity of $v=0.9$, we will have $0.1(4000-100)=390$ false detections. Figures 5(a) and (b) show the effects of non-ideal specificity of the detectors on the mean and standard 

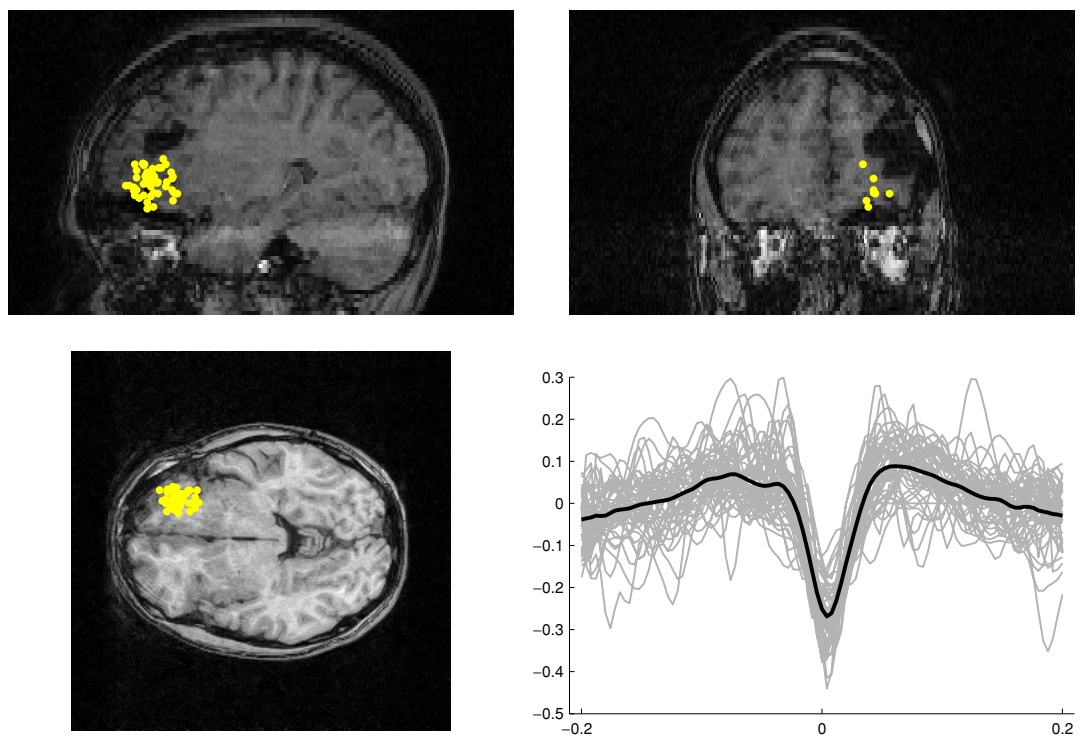

(c)

Figure 6. (Continued.)

deviation of the transition probability estimate. The mean of the estimate of a non-zero-valued element $p\left(E_{1} \mid P_{2}\right)$ acquires some multiplicative bias. The mean of the estimate of a zerovalued element $p\left(E_{2} \mid P_{1}\right)$ increases as the FAR grows. The tendency is that, for very high FAR (several hundred false detections per minute!), the contrast between the estimates of zero and non-zero transition probability values disappears and propagation detection becomes impossible. Fortunately, a FAR of several hundred false detections per minute is unrealistically high.

The results of taking into consideration non-ideal specificity $v$ of the detectors can be analysed by comparing figures 5(a) and (b) with (c) and (d), where we now incorporate the true specificity-sensitivity characteristics. One of the effects of accounting for the detector specificity is that the contrast between the estimates of non-zero-valued $\hat{p}\left(E_{1} \mid P_{2}\right)$ and zero-valued $\hat{p}\left(E_{2} \mid P_{1}\right)$ increases which allows for better detection. The undesired consequence of taking into account the characteristics of the detector is that the standard deviation of the estimate increases.

We therefore conclude that accounting for imperfect detection allows us to obtain improved estimates of the transition probability matrix. As we have described in section 2.3, the detector specificity and sensitivity can be incorporated into the model via modification of the state emission probability functions according to equations (2) and (3). The inevitable consequence of introducing this additional uncertainty into the model is the increase of standard deviation of the estimate.

\section{Application to real interictal MEG data}

In a second paper under preparation, we more thoroughly investigate the application of this method to several clinical data sets. Here, we present briefly four independently recorded data sets from a single patient as an example. Operating on the principles described above, we applied a prototype version of the propagation analysis software to MEG data obtained 

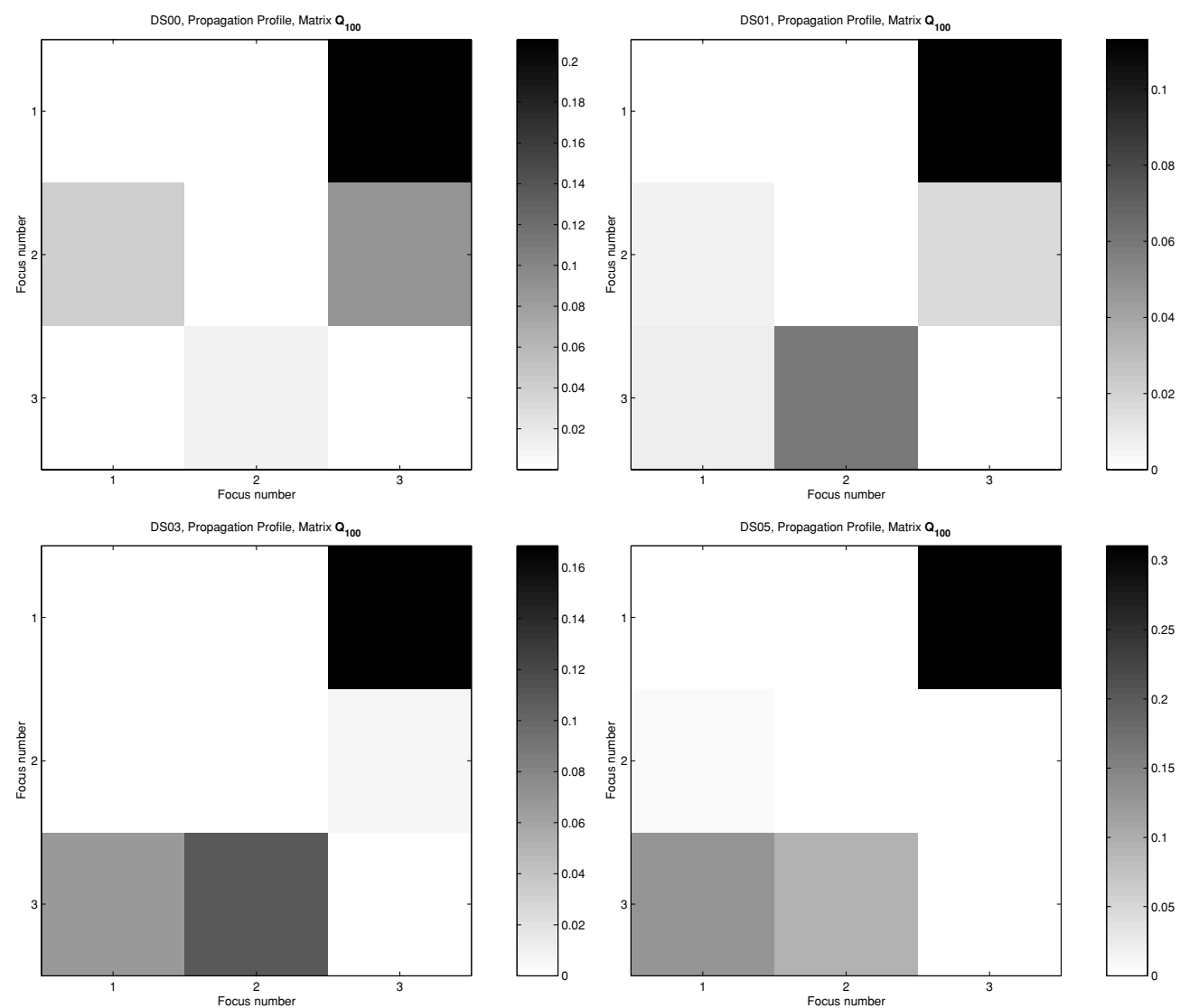

Figure 7. Propagation pattern represented using matrix $\mathbf{Q}^{100 \mathrm{~ms}}$ for the four independently collected data sets.

from one patient with multifocal partial epilepsy recorded using a 68-channel CTF MEG machine. We applied our automatic spike detection technique (Ossadtchi et al 2004) followed by propagation detection to four independently collected interictal MEG data sets from this patient.

For each data set, three major clusters were found, as shown in figure 6 . The first cluster was located in the right temporal lobe, the second in the superior frontal lobe and the third cluster was in the inferior mesial frontal lobe area. In order to assess the propagation of the interictal activity, we applied our propagation detection method to each of the four data sets using a three-focus model. The resulting HMM consisted of 7 states and 19 allowed transitions. For brevity in this example, we did not perform analysis of the detection performance but rather assumed nearly ideal specificity and sensitivity. To ensure convergence of the EM algorithm, we assumed a specificity for all three detectors of $\nu=0.999$ and a sensitivity of $\mu=0.999$.

In figure 7, we show grey-scale-coded $\rho=\left\{\rho_{i j}\right\}$ matrices, computed from the estimated transition probability matrix using (1) for $\tau_{0}=4 \mathrm{~ms}$ and $\tau_{1}=100 \mathrm{~ms}$. The $(i, j)$ th element of the $\rho$-matrix corresponds to the probability of propagation from the $i$ th focus to the $j$ th focus within the time window of $96 \mathrm{~ms}$ starting at $4 \mathrm{~ms}$ after the initial spike, as described in section 2.3. The four propagation probability matrices computed from the four independent data sets are shown in figure 7 . 
All four matrices indicate a relatively high probability of propagation from cluster 1 (right lateral temporal lobe) to cluster 3 (right inferior frontal lobe). The propagation occurred within a plausible time interval of $4 \mathrm{~ms}$ and $96 \mathrm{~ms}$ following the initial spike cluster 1 . The last two data sets, in addition to propagation from the temporal lobe to the frontal lobe, also show a significant amount of propagation activity from the frontal lobe back to the temporal lobe and from the fontal lobe to the superior mesial frontal lobe; however, the intensity of this propagation is less than that from the temporal to the fontal lobe. A subsequent surgery in which this portion of the temporal lobe (as well as a portion of the frontal lobe) was removed has left the patient seizure free for already more than 48 months.

\section{Discussion and conclusions}

We have described a procedure for the detection of propagation between several brain regions during interictal activity. Our method employs the HMM framework for modelling the outputs of the spike detectors tuned to each of several brain regions. We have shown how the parameters of the HMM transition probability matrix can be translated into the physiological properties of propagation activity. The identification of the HMM transition probability matrix has been performed using a constrained version of the classical Baum-Welch EM algorithm. We have also studied the potential accuracy of the estimates by deriving an analytical expression for the lower bound on the variance of the transition probability estimates.

The HMM paradigm allows us to model the observation process by including the specificity-sensitivity characteristics of the detectors used for identification of spike events in the selected regions. We have performed Monte Carlo simulations in order to investigate the effects of non-ideal detector characteristics and the amount of improvement that can be achieved by including the detector properties in the model. The simulations demonstrate the robustness of the method to the non-ideal specificity and sensitivity characteristics. This is particularly important since our ultimate goal is to apply these methods to clinically obtained non-invasive MEG data, and use the resulting analysis as a diagnostic aid.

Our work has employed classical HMMs. One potential problem with the classical HMM is that the distribution of the time spent in a state is geometric. This contradicts the intuitively expected unimodal (e.g., $\chi^{2}$ ) distribution of propagation state duration centred at a characteristic delay. This problem can be overcome by the use of variable state duration HM models (Levinson 1986). However, it can be shown that for a fixed training data set the off-diagonal elements of the classical HMM transition probability matrix will have the same values as for the variable state duration HMM fitted to the same data. In addition, the fitting process for the variable state duration HMM is significantly less stable then fitting the classical HMM. The ultimate goal of propagation analysis is to identify the primary focus that triggers abnormal activity in other regions, and our simulation studies are consistent with the conclusion that classical HMMs are sufficient for this purpose. With simulated data, we have demonstrated that analysis of the transition probability matrix of the classical HMM allows for a robust determination of the primary focus, even as sensitivity deteriorates.

By combining the HMM methods described in this paper with the automatic spike identification and clustering methods described in Ossadtchi et al (2004), we have laid the algorithmic foundation for a method that can be used automatically to scan large data sets and classify brain regions to identify candidate epileptogenic zones from extracranial data. Complex spikes or trains of spikes may be decomposed into their individual components, and the resulting analysis may yield a decomposition and temporal ordering not apparent from visual inspection of the data alone. The approach also allows us to include explicitly 
the uncertainties associated with non-ideal detector sensitivity and specificity. The resulting analysis is suitable for non-parametric statistical significance testing.

In a preliminary study (Ossadtchi et al 2003), and in the brief example here, we have applied these methods to four independent interictal data sets collected from a patient with multifocal epilepsy. Three epileptogenic regions were automatically detected and the propagation detection technique described here was applied. In all four data sets, propagation from the temporal lobe to the frontal lobe was detected reliably and was clearly dominant. A more detailed evaluation of the HMM in application to clinical data, including permutation tests to determine statistical significance, will be presented in a companion paper.

\section{Acknowledgments}

This work was supported by the National Institutes of Health under grants R01-EB002010, R01-NS020806, R43-NS051056 and by Los Alamos National Laboratory, operated by the University of California for the United States Department of Energy, under contract W-7405ENG-36.

\section{Appendix. Theoretical estimator and variance of the State transition probability matrix estimate}

For compact expressions, we replace the bracketed indices for the HMM parameters with the subscripts that index elements in the set $s \in\left\{I, P_{1}, \ldots, P_{N_{f}}, E_{1}, \ldots, E_{N_{f}}\right\}$. As an example, elements of the transition probability matrix $p(s=j \mid s=i)$ will be denoted as $p_{i j}$.

\section{A.1. MVU estimator in the non-hidden case}

We determine the MVU estimator based on the notion of sufficient statistics. The parameters to be estimated are the elements $p_{i j}$ of the $N_{s} \times N_{s}$ transition probability matrix $\mathbf{P}$.

We first group the unknown parameters into $N_{s}$ vectors $\mathbf{p}_{i}=\left[p_{i 1}, \ldots, p_{i N_{s}}\right], i=$ $1, \ldots N_{s}$. Then, for the $k$ th vector $\mathbf{p}_{k}$ the vector $\mathbf{T}$ of complete sufficient statistics can be found based on the Neyman-Fisher factorization theorem as follows:

$$
\begin{aligned}
p(\mathcal{Z} ; \mathbf{P} ; \boldsymbol{\pi}) & =\exp \left(\sum_{t=0}^{T-2} \sum_{i=1}^{N_{s}} \sum_{j=1}^{N_{s}} \ln \left(p_{i j}\right) z_{t}^{i} z_{t+1}^{j}+\sum_{i=1}^{N_{s}} \pi_{i} z_{1}^{i}\right) \\
& =\exp \left(\sum_{j=1}^{N_{s}} \log p_{k j} \sum_{t=0}^{T-2} z_{t}^{k} z_{t+1}^{j}\right) \times \exp \left(\sum_{i=1, i \neq k}^{N_{s}} \sum_{j=1}^{N_{s}} \log p_{i j} \sum_{t=0}^{T-2} z_{t}^{i} z_{t+1}^{j}+\sum_{i=1}^{N_{s}} \pi_{i} z_{1}^{i}\right) \\
& =\exp \left(\sum_{j=1}^{N_{s}} \log p_{k j} \chi_{k j}(\mathcal{Z})\right) \times \exp \left(\sum_{i=1, i \neq k}^{N_{s}} \sum_{j=1}^{N_{s}} \log p_{i j} \chi_{i j}(\mathcal{Z})+\sum_{i=1}^{N_{s}} \pi_{i} z_{1}^{i}\right) \\
& =\exp \left(\chi_{k}^{T} \log \mathbf{p}_{k}\right) \times \exp \left(\sum_{i=1, i \neq k}^{N_{s}} \chi_{i}^{T} \log \mathbf{p}_{i}+\sum_{i=1}^{N_{s}} \pi_{i} z_{1}^{i}\right) \\
& =g\left(\chi_{k}, \mathbf{p}_{k}\right) \times h(\mathcal{Z}) .
\end{aligned}
$$

According to the Neyman-Fisher factorization theorem, $\chi_{k}$ with elements

$$
\chi_{k j}=\sum_{t=0}^{T-2} z_{t}^{k} z_{t+1}^{j}
$$


is a sufficient statistic for estimating the $k$ th vector of transition probabilities $\mathbf{p}_{k}$. The following transformation turns sufficient statistics vector $\chi_{k}$ into an unbiased estimator of the vector $\mathbf{p}_{k}$ :

$$
\hat{\mathbf{p}}_{k}=\frac{\chi_{k}}{\mathbf{1}^{T} \chi_{k}}
$$

where 1 is a $N_{s} \times 1$ vector of ones.

Inspection of equation (A.2) and binary nature of the indicator vectors allow to conclude that

$$
\hat{p}_{k j}=\frac{N_{k j}}{N_{k}}
$$

which is in agreement with (13), where $N_{k j} \equiv N(j \mid k)$, i.e. the number of transitions from focus $k$ to focus $j$. To check that equation (13) is indeed an unbiased estimator, we first specify the expression for the joint PDF $p\left(N_{i j}, N_{i}\right)$ in terms of quantities $N_{i j}$ and $N_{i}$. It can be obtained as a product of the conditional PDF $p\left(N_{i j} \mid N_{i}\right)$ and marginal PDF $p\left(N_{i}\right)$, i.e.

$$
\begin{aligned}
& p\left(N_{i j}, N_{i}\right)=p\left(N_{i j} \mid N_{i}\right) p\left(N_{i}\right), \\
& p\left(N_{i j} \mid N_{i}\right)=\left(\begin{array}{c}
N_{i} \\
N_{i j}
\end{array}\right) p_{i j}^{N_{i j}}\left(1-p_{i j}\right)^{N_{i}-N_{i j}}, \\
& p\left(N_{i}\right)=\left(\begin{array}{c}
T \\
N_{i}
\end{array}\right) \pi_{i}^{N_{i}}\left(1-\pi_{i}\right)^{T-N_{i}} .
\end{aligned}
$$

Then we compute the expectation of the $N_{i j} / N_{i}$ ratio as

$$
\begin{aligned}
E\left\{\hat{p}_{i j}\right\} & =E\left\{\frac{N_{i j}}{N_{i}}\right\}=\sum_{N_{i}=1}^{T} \frac{1}{N_{i}} p\left(N_{i}\right) \sum_{N_{i j}=1}^{N_{i}} N_{i j} p\left(N_{i j} \mid N_{i}\right) \\
& =p_{i j} \sum_{N_{i}=1}^{T} p\left(N_{i}\right)=p_{i j},
\end{aligned}
$$

and conclude that the estimator (13) is indeed unbiased.

The PDF (A.1) belongs to the family of vector exponential PDFs as it can be written as

$$
f\left(\mathcal{Z}, \mathbf{p}_{k}\right)=h(\mathcal{Z}) C\left(\mathbf{p}_{i}\right) \exp \left(\sum_{i=1}^{N_{s}} \log p_{k i} \chi_{k i}\right)
$$

meaning that the sufficient statistics $\chi_{k}$ is complete (Kendall and Stuart 1979). The completeness of the sufficient statistics ensures that the estimator (A.2) is the minimum variance estimator.

\section{A.2. Variance of the MVU estimator in the non-hidden case}

The variance of the transition probability estimator $\hat{p}_{i j}=N_{i j} / N_{i}$ can be computed as the following difference:

$$
\begin{aligned}
\operatorname{var}\left\{\frac{N_{i j}}{N_{i}}\right\} & =E\left\{\frac{N_{i j}^{2}}{N_{i}^{2}}\right\}-E^{2}\left\{\frac{N_{i j}}{N_{i}}\right\} . \\
\operatorname{var}\left\{\frac{N_{i j}}{N_{i}}\right\} & =\sum_{N_{i}=1}^{T} \frac{1}{N_{i}^{2}} p\left(N_{i}\right) \sum_{N_{i j}=1}^{N_{i}} N_{i j}^{2} p\left(N_{i j} \mid N_{i}\right)-\sum_{N_{i}=1}^{T} \frac{1}{N_{i}} p\left(N_{i}\right) \sum_{N_{i j}=1}^{N_{i}} N_{i j} p\left(N_{i j} \mid N_{i}\right) \\
& =p_{i j}\left(1-p_{i j}\right) \sum_{N_{i}=1}^{T} \frac{1}{N_{i}}\left(\begin{array}{c}
T \\
N_{i}
\end{array}\right) \eta^{N_{i}}\left(1-\eta_{i}\right)^{T-N_{i}} .
\end{aligned}
$$


In order to obtain a useful approximation we expand $1 / N_{i}$ around its mean value of $E\left\{N_{i}\right\}=T \eta_{i}$. Then we compute expectation for each term in the expansion and get the following:

$\operatorname{var}\left\{\frac{N_{i j}}{N_{i}}\right\}=p_{i j}\left(1-p_{i j}\right)\left(\frac{1}{T \eta_{i}}+\frac{1-\eta_{i}}{T^{2} \eta_{i}^{2}}-\frac{\left(1-\eta_{i}\right)\left(1-2 \eta_{i}\right)}{T^{3} \eta_{i}^{3}} \cdots\right)$.

As $T$ is usually large, on the order of $10^{5}$, keeping only the first two members of the expansion delivers reasonable accuracy. Thus, we conclude that

$$
\operatorname{var}\left\{\frac{N_{i j}}{N_{i}}\right\}=p_{i j}\left(1-p_{i j}\right)\left(\frac{1}{T \eta_{i}}+\frac{1-\eta_{i}}{T^{2} \eta_{i}^{2}}\right)+o\left(\frac{1}{\eta_{i} T^{3}}\right) .
$$

\section{References}

Baumgatner C, Lindinger G, Ebner A, Aull S, Serles W, Olbrich A, Lurger S, Czech T, Burgess R and Luders H 1995 Propagation of interictal activity in temporal lobe epilepsy Neurology 45 118-22

Bourien J, Bellanger J, Bartolomei F, Chauvel P and Wendling F 2004 Mining reproducible activation patterns in epileptic intracerebral EEG signals: application to interictal activity IEEE Trans. Biomed. Eng. 512

Diekmann V, Becker W, Jurgens R, Grozinger B, Kleiser B, Richter H and Wollinsky K 1998 Localisation of epileptic foci with electric, magnetic and combined electromagnetic models Electroencephalogr. Clin. Neurophysiol. 106 297-313

Duckrow R B and Spencer S S 1998 Regional coherence and the transfer of ictal activity during seizure onset in the medial temporal lobe Electroencephalogr. Clin. Neurophysiol. 42 415-22

Franaszczuk P J and Bergey G K 1999 An autoregressive method for the measurement of synchronization of interictal and ictal EEG signals Biol. Cybern. 81 3-9

Gloor P 1985 Neuronal generators and the problem of localization in electroencephalography: application of volume conductor theory to electroencephalography J. Clin. Neurophysiol. 2 327-54

Kaminski M and Blinowska. K 1991 A new method of the description of the information flow in the brain structures Biol. Cybern. 65 203-10

Kay S 1988 Computer applications Modern Spectral Analysis (Englewood Cliffs, NJ: Prentice-Hall)

Kay S 1993 Cramer-Rao lower bound Fundamentals of Statistical Signal Processing: Estimation Theory (Englewood Cliffs, NJ: Prentice-Hall)

Kendall S M and Stuart A 1979 The Advanced Theory of Statistics vol 2 (New York: Macmillan)

Kobayashi K, Akiyama T and Nakahori T 2002 Systematic source estimation of spikes by a combination of independent component analysis and RAP-MUSIC: II. Preliminary clinical application Clin. Neurophisiol. 113 725-34

Kobayashi K, James C, Nakahori T, Akiyama T and Gotman J 1999 Isolation of epileptiform discharges from unaveraged EEG by independent component analysis Clin. Neurophisiol. 110 1755-63

Kudela P, Franaszczuk P and Bergey G 1999 Model of the propagation of synchronous firing in a reduced neuron network Neurocomputing 26-27 411-8

Levinson S 1986 Continuously variable duration hidden Markov model for automatic speech recognition Comput. Speech Lang. $129-45$

McIntosh A, Bookstein F and Grady J H C 1996 Spatial pattern analysis of functional brain images using partial least squares Neuroimage 3 143-57

Moradi F 2004 Information coding and oscillatory activity in synfire neural networks with and without inhibitory coupling Biol. Cybern. 91 283-94

Nunez P L, Silberstein R B, Shi Z, Carpenter M R, Srinivasan R, Tucker D M, Duran S M, Cadusch P J and Wijesinghe R S 1999 EEG coherency II: experimental comparisons of multiple measures Clin. Neurophysiol. 110 469-86

Ossadtchi A, Mosher J, Jerbi K, Lopez N, Baillet S, Sutherling W and Leahy R 2004 Automated interictal spike detection and source localization in magnetoencephalography using independent component analysis and spatiotemporal clustering Clin. Neurophysiol. 115 508-22

Ossadtchi A, Mosher J, Sutherling W and Leahy R 2003 Non-invasive HMM-based detection of interictal activity propagation between several epileptogenic foci in patients with multi-focal epilepsy Epilepsia 44 (Suppl. 9) 232 
Rabiner L and Juang B 1986 An introduction to hidden Markov models IEEE ASSP Mag. 1 4-15

Rabiner L and Juang B 1993 Fundamentals of Speech Recognition (Englewood Cliffs, NJ: Prentice-Hall) chapter 1

Strother S et al 1996 Multidimensional state-spaces for fMRI and pet activation studies Neuroimage 3 S 98

Sutherling W and Barth D 1989 Neocortical propagation in temporal lobe spike foci on magnetoencephalography and encephalography Ann. Neurol. 25 373-81

Tuckwell H 1988 Introduction to Theoretic Neurobiology (Cambridge: Cambridge University Press) 